\title{
N1-Guanyl-1,7-Diaminoheptane Sensitizes Estrogen Receptor Negative Breast Cancer Cells to Doxorubicin by Preventing Epithelial-Mesenchymal Transition through Inhibition of Eukaryotic Translation Initiation Factor 5A2 Activation
}

\author{
Yu Liu ${ }^{a, b}$ Rongrong Liu ${ }^{c}$ Peifen Fu ${ }^{a, b}$ Feiya Du ${ }^{d}$ Yun Hong ${ }^{a, b}$ Minya Yao a,b \\ Xianning Zhang ${ }^{c}$ Shusen Zheng ${ }^{a, b}$
}

\begin{abstract}
${ }^{a}$ Department of General Surgery, ${ }^{b}$ Key Laboratory of Combined Multi-organ Transplantation, Ministry of Public Health, First Affiliated Hospital, School of Medicine, Zhejiang University, Hangzhou, 'Department of Cell Biology and Medical Genetics, Research Center of Molecular Medicine, National Education Base for Basic Medical Sciences, Institute of Cell Biology, Zhejiang University School of Medicine, Hangzhou, Zhejiang Province, dDepartment of Orthopaedics, First Affiliated Hospital, School of Medicine, Zhejiang University, Hangzhou, China
\end{abstract}

\section{Key Words}

Breast cancer • Estrogen receptor • Epithelial-mesenchymal transition • eIF5A2

\begin{abstract}
Background: Approximately $30 \%$ of breast cancer does not express the estrogen receptor $(E R)$, which is necessary for endocrine-based therapy approaches. Many studies demonstrated that eukaryotic translation initiation factor 5A2 (eIF5A2) serves as a proliferation-related oncogene in tumorigenic processes. Methods: The present study used cell viability assays, EdU incorporation assays, western blot, and immunofluorescence to explore whether N1guanyl-1,7-diaminoheptane (GC7), which inhibits eIF5A2 activation, exerts synergistic cytotoxicity with doxorubicin in breast cancer. Results: We found that GC7 enhanced doxorubicin cytotoxicity in ER-negative HCC1937 cells but had little effect in ER-positive MCF-7 and Bcap-37 cells. Administration of GC7 reversed the doxorubicin-induced epithelialmesenchymal transition (EMT) in ER-negative breast cancer cells. Knockdown of eIF5A2 by siRNA inhibited the doxorubicin-induced EMT in ER-negative HCC1937 cells. Conclusion: These data demonstrated that GC7 combination therapy may enhance the therapeutic efficacy of doxorubicin in estrogen negative breast cancer cells by preventing EMT through inhibition of eIF5A2 activation.

Y. Liu and R. Liu contribute equally to this article.

Shusen Zheng, PhD,

Xianning Zhang, PhD

KARGER 125

Department of General Surgery, The First Affiliated Hospital, School of Medicine,

Zhejiang University, 79 Qinchun Road, Hangzhou (China); Department of Cell

Biology and Medical Genetics, Research Center of Molecular Medicine, National

Education Base for Basic Medical Sciences, Institute of Cell Biology, Zhejiang University

School of Medicine, Hangzhou, Zhejiang Province 310058 (China)

E-Mail shusenzheng@zju.edu.cn, E-Mail zhangxianing@zju.edu.cn
\end{abstract}




\section{Introduction}

Breast cancer has emerged as the most common malignancy observed in females worldwide. It has become the leading cause of cancer death among females, accounting for $23 \%$ of the total cancer cases and $14 \%$ of the cancer deaths $[1,2]$. Estrogen is an important regulator for growth and differentiation of the normal mammary gland as well as the malignant progression of about $70 \%$ of breast cancer. It exerts its biological effects through binding to the estrogen receptor (ER) which is a transcription factor and controls cell proliferation and differentiation $[3,4]$. Like other steroid hormone receptors, the ER modulates its downstream effects by transcriptional regulation of target genes. During the past years, targeting the ER has been the cornerstone for the treatment of ER-positive (ER $(+)$ ) breast cancers [5]. A recent study suggests that short-term estradiol treatment confers protection against breast cancer by targeting major cell signaling pathways such as JAK2 and STATs [6]. However, approximately $30 \%$ of all breast cancers lack detectable ER protein (ER) and are rarely responsive to hormonal treatment with a more aggressive clinical course [7]. Therefore, it is urgent to develop new and effective targeted therapies for ER (-) breast cancer.

It is generally believed that tumorigenesis in the breast cancer is a multistep process regulated by aberrantly protein expression and alterations of morphological and molecular features during malignant progression [8-10]. One such change is the loss of epithelial properties, which suggests the initiation of epithelial-mesenchymal transition (EMT). Increasing evidences demonstrate EMT has been recognized as a critical procedure regulating the chemoresistance properties of breast cancer [11].

Eukaryotic translation initiation factor 5A2 (eIF5A2), one isoform of eIF5A, has been identified as a novel oncogene in ovarian cancer [12]. A recent study examined the eIF-5A2 expression and doxorubicin sensitivity in human breast cancer cell lines and demonstrated that eIF-5A2 played an important role in doxorubicin chemoresistance in breast cancer [13]. Inhibition of elF5A2 by N1-guanyl-1, 7-diaminoheptane (GC7), an inhibitor of deoxyhypusin synthase (DHS), exerts obvious anti-tumor effects in human cancer cells [14]. Moreover, numerous studies demonstrated that eIF5A2 exerted great impact on EMT progression in many cancers by transcriptional regulation of different downstream molecules, such as hepatocellular carcinoma, colorectal carcinoma and bladder cancer $[15,16]$. However, the relationship between eIF5A2 and EMT in breast cancer cells has not been investigated. Therefore, in the present study, we examined the anti-tumor effects of doxorubicin-based treatment combined with GC7 in breast cancer cells. In addition, we explored the underlying mechanisms of the combined therapy, and showed that eIF5A2 inactivation induced by GC7 suppressed doxorubicin-induced EMT in breast cancer cells.

\section{Materials and Methods}

Cell culture and reagents

Human breast cancer cell lines MCF-7, HCC1937, and Bcap-37 were purchased from the ATCC (Manassas, VA, USA) and cultured in DMEM (Gibco, Carlsbad, CA, USA) supplemented with 10\% FBS and 1\% penicillin/streptomycin. All cells were maintained at $37^{\circ} \mathrm{C}$ in $5 \% \mathrm{CO} 2$ incubator. Doxorubicin and GC7 were purchased from Sigma-Aldrich (St. Louis, MO, USA). The eIF5A2 siRNA and negative control siRNA were purchased from Santa Cruz Biotechnology (Santa Cruz, CA, USA).

CCK-8 assay

Breast cancer cells or siRNA-transfected breast cancer cells were seeded onto 96well plates at 3000 cells/well. The medium was replaced with the corresponding serum-free medium for $24 \mathrm{~h}$ to synchronize the cell cycle, then serum-free medium was replaced with complete medium containing the drugs at the indicated concentrations for $48 \mathrm{~h}$. Then $10 \mu \mathrm{L} /$ well CCK8 solution (Dojindo, Kumamoto, Japan) was added, the plates incubated for $3 \mathrm{~h}$, and absorbance was measured at $450 \mathrm{~nm}$ using an MRX II microplate reader (Dynex, Chantilly, VA, USA). 
EdU incorporation assay

Cell viability was calculated as a percentage of untreated control. Measurement of inhibitive rate of cell proliferation was carried out using a Click-iT EdU Imaging Kit (Invitrogen, Carlsbad , CA, USA) following the procedure previously described [17].

\section{SiRNA transfection}

Cells were transfected with eIF5A2 siRNA or negative control siRNA using Lipofectamine 2000 (Invitrogen) according to the manufacturer's protocol. The transfection medium (Opti-MEM; Gibco ) was replaced with complete medium $12 \mathrm{~h}$ after transfection, and the cells were incubated for the indicated times.

\section{Western blot analysis}

Breast cancer cells were lysed in $50 \mu \mathrm{l}$ cell lysis buffer ( Cell Signaling, Danvers, MA, USA) containing protease inhibitors (Sigma). The protein concentration was quantified using the BCA Protein Kit (Thermo, Rockford, IL, USA). Cell lysates were separated by 10\% SDS-PAGE and proteins were transferred to polyvinylidene difluoride (PVDF) membranes (Millipore, Billerica, MA, USA). The membranes were then incubated with primary antibodies (E-cadherin, Vimentin or eIF5A2, diluted 1:1000; Abcam, Cambridge, USA) at $4^{\circ} \mathrm{C}$ overnight. The membranes were washed three times with TBS/T and then incubated with the appropriate HRP-conjugated secondary antibodies for $1 \mathrm{~h}$ at room temperature. Protein expression was detected by chemiluminescence (GE Healthcare, Piscataway, NJ, USA).

Immunofluorescence

Breast cancer cells or siRNA-transfected cells were seeded into 48-well plates at 6000 cells/well. Cells were fixed with $4 \%$ formaldehyde for $15 \mathrm{~min}$, washed with PBS, treated with 5\% BSA for 30 min at room temperature, and incubated with mouse anti-human vimentin or anti-human E-cadherin primary antibodies (Cell Signaling Technology) at $4^{\circ} \mathrm{C}$ overnight. The cells were incubated with goat anti-mouse FITC-conjugated secondary antibody (Abcam) at $4{ }^{\circ} \mathrm{C}$ for $2 \mathrm{~h}$, incubated with DAPI (Sigma-Aldrich) for 2 min at room temperature, washed twice with PBS, and observed using an inverted fluorescence microscope (Olympus, Tokyo, Japan).

\section{Measurement of eIF5A2 activity}

Formation of hypusinated eIF5A2 is essential for eIF5A2 maturation. The activity of eIF5A2 was measured by counting the radioactivity of ${ }^{3} \mathrm{H}$-labelled spermidine. In brief, breast cancer cells were incubated with $\left[1,8{ }^{3} \mathrm{H}\right]$-spermidine $(10 \mu \mathrm{Ci} / \mathrm{mL}$; Perkin-Elmer/NEN, Boston, MA, USA) for $48 \mathrm{~h}$. Cells were precipitated in $10 \%$ trichloroacetic acid containing $1 \mathrm{mM}$ unlabeled spermidine and spermine and washed three times. The trichloroacetic acid precipitate was used for SDS-PAGE and the radioactivity of hypusinated eIF5A2 was determined by fluorography.

\section{Statistical analysis}

Data were presented as the mean \pm SD. Statistical analysis was carried out using PRISM 5 (GraphPad, San Diego, CA, USA). The effects of combined treatment were compared using two-way ANOVA, followed by Bonferroni's post-hoc test. Analyses for two groups comparing were carried out using Student's $\mathrm{t}$-tests. A $P$-value $<0.05$ was considered statistically significant.

\section{Results}

Low concentrations of GC7 have little effect on breast cancer cell viability

Firstly, CCK-8 assay was performed to determine the appropriate concentration of GC7 for combined treatment with doxorubicin. A series of GC7 concentrations ranging from 0 50 $\mu \mathrm{M}$ were incubated with three breast cells lines (HCC1937, Bcap-37 and MCF-7) and data from CCK-8 assay showed that GC7 exerted little cytotoxicity in cancer cells between 0 and $5 \mu \mathrm{M}$. However, higher concentrations of GC7 $(10,20,50 \mu \mathrm{M})$ significantly inhibited the viability of the three cell lines (Fig. 1A-C). Therefore, $5 \mu \mathrm{M}$ GC7 was used for further 
coadministration with doxorubicin. To confirm the status of ER expression, western blot was used to detect the ER expression in three different cell lines. Results showed that HCC1937 was ER-negative, while MCF-7 and Bcap-37 were ER-positive (Fig. 1D).

GC7 enhanced the cytotoxicity of doxorubicin in ER-negative breast cancer cells

To evaluate the synergistic cytotoxic effect of doxorubicin combined with GC7, we used CCK-8 assay to measure cell viability treated for $48 \mathrm{~h}$ with doxorubicin alone or doxorubicin plus GC7. ER-negative HCC1937 cells showed a higher sensitivity to doxorubicin in the presence of GC7. In contrast, no significant change in cytotoxicity of doxorubicin has been observed in ER-positive MCF-7 and Bcap-37 cells with or without GC7 (Fig. 2A-C). Furthermore, EdU incorporation assay was used to test the inhibition of cells proliferation after treatment for $48 \mathrm{~h}$ with doxorubicin alone or doxorubicin plus GC7. Consistent with data in CCK-8 assay, GC7 obviously significantly decreased the cell proliferation in ER-negative HCC1937 cells other than ER-positive MCF-7 and Bcap-37 cells (Fig. 2D-F). Collectively, these data indicated that GC7 could enhance the sensitivity of ER-negative breast cancer cells to doxorubicin.

The doxorubicin-induced EMT can be reversed by GC7 in ER-negative breast cancer cells

In order to investigate whether doxorubicin can induce EMT in breast cancer cells, we detected the expression of epithelial / mesenchymal markers in breast cancer cells treated with doxorubicin for $48 \mathrm{~h}$. As shown in Figure 3A, administration of doxorubicin significantly decreased the expression of E-cadherin, and enhanced the expression of Vimentin in HCC1937, MCF-7 and Bcap-37 cells. Interestingly, the expression of E-cadherin and Vimentin was reversed only in ER-negative HCC1937 treated with doxorubicin plus GC7, indicated that GC7 reversed the doxorubicin-induced EMT in ER-negative HCC1937 cells other than ER-positive MCF-7 and Bcap-37 cells (Fig. 3B-D). Moreover, immunofluorescent staining also showed similar results which were consistent with the western blotting (Fig. 3E).

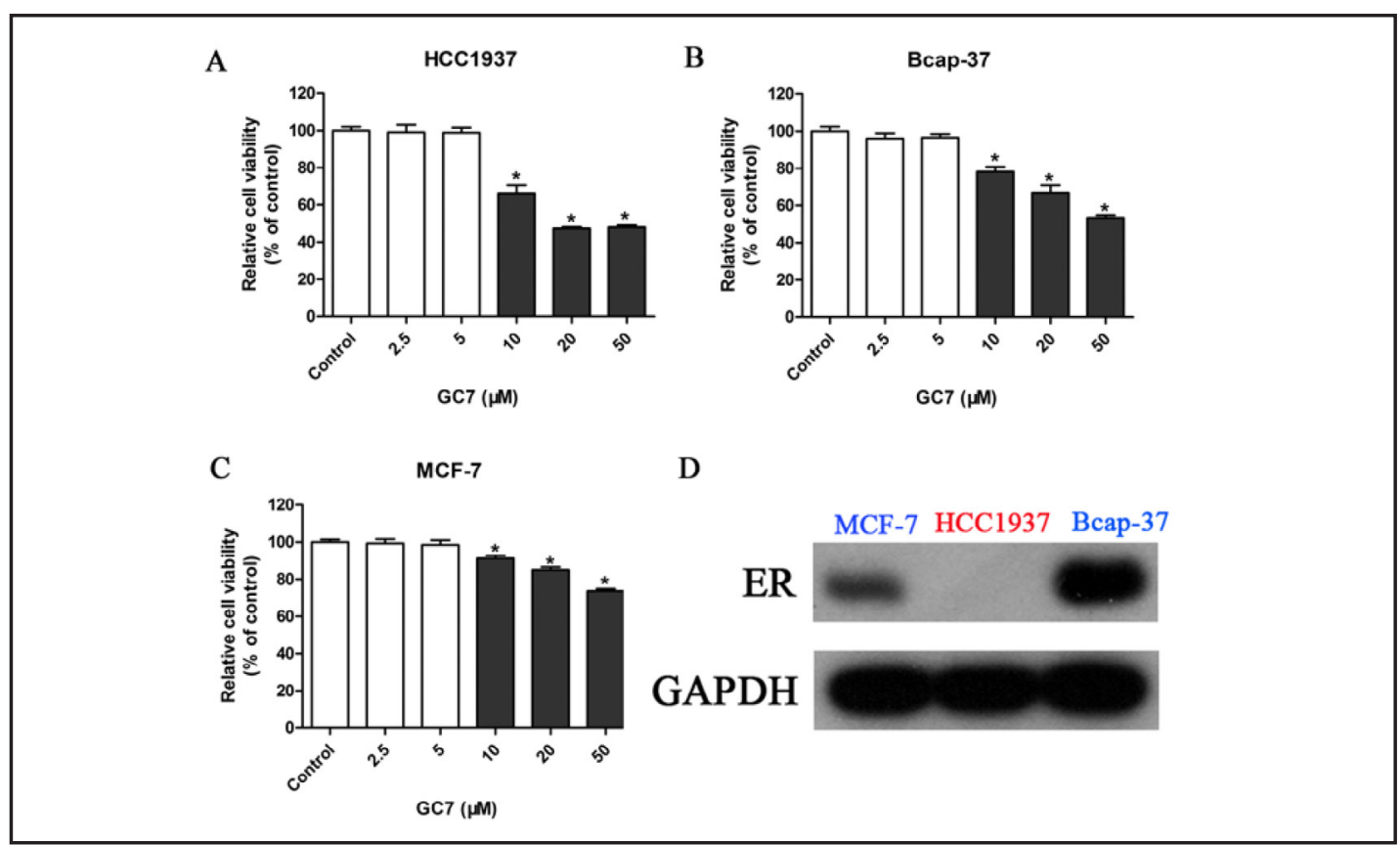

Fig. 1. Determination of the effect of GC7 on cytotoxicity and expression of ER in breast cancer cells. Three breast cancer cell lines including HCC1937 (A), Bcap-37 (B) and MCF-7 (C) were incubated with different concentrations of GC7 for $48 \mathrm{~h}$. The CCK8 values of the treated breast cancer cells were normalized to the control group with the absence of GC7. ${ }^{*} \mathrm{P}<0.05$. (D) Western blot was used to detect the ER expression in three different cell lines. All experiments were performed at least three times. 


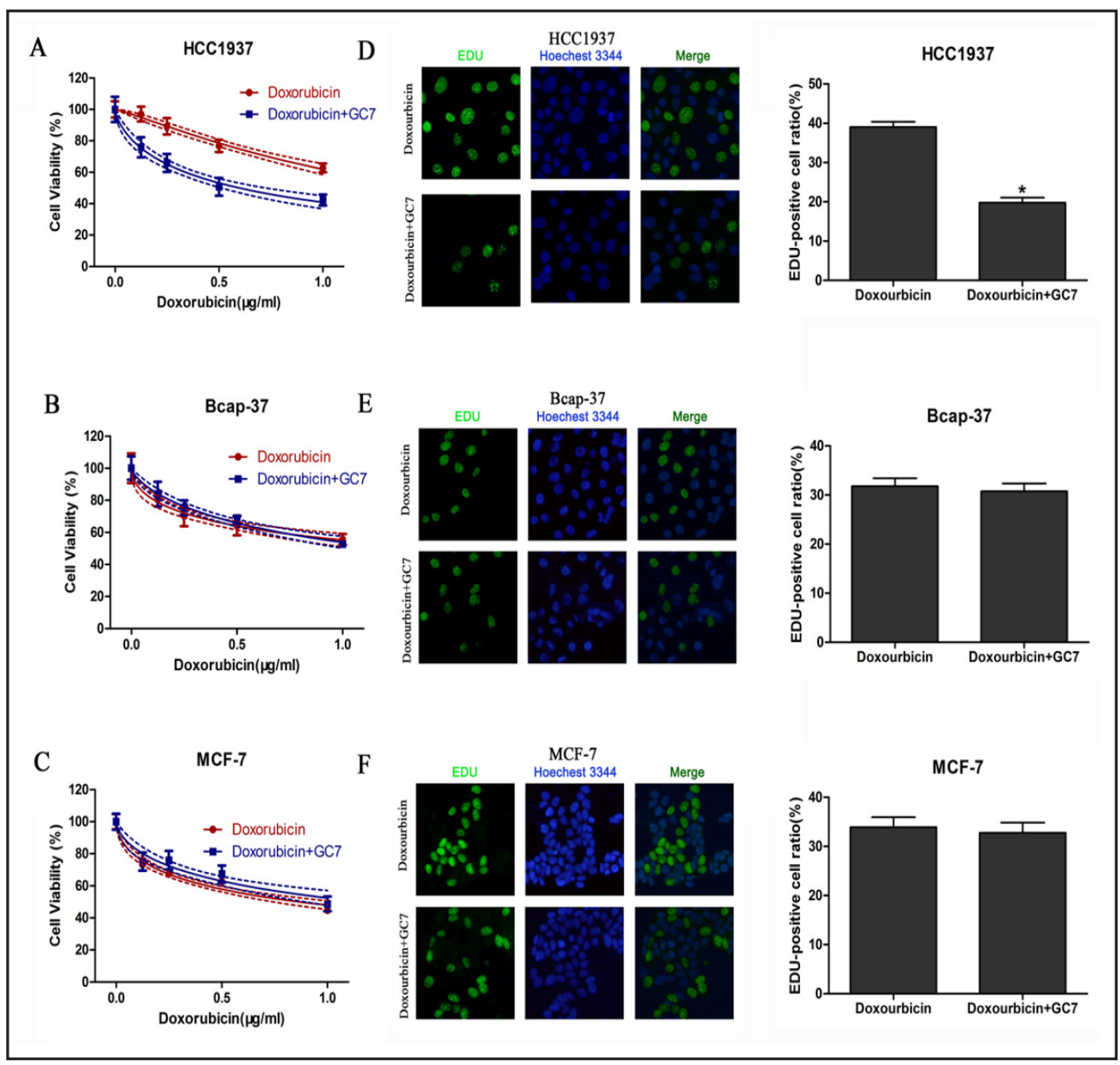

Fig. 2. Cytotoxicity of doxorubicin or doxorubicin plus GC7 in breast cancer cells. GC7 (5 $\mu \mathrm{M})$ significantly enhanced the cytotoxicity of doxorubicin in HCC1937 (A), Bcap-37 (B) and MCF-7 (C) cell. Photomicrographs and bar charts depict the EdU staining and relative EdU-positive ratio, respectively, of HCC1937 (D), Bcap37 (E) and MCF-7 (F) cell after treatment with doxorubicin or doxorubicin plus GC7 for 48 h. * P $<0.05$. All experiments were performed at least three times.

Knockdown of eIF5A2 diminished the doxorubicin-induced EMT in ER-negative HCC1937 cells

Deoxyhypusine synthase is specifically inhibited by GC7 in mammalian cells, which catalyzes the post-translation modifications required to activate eIF5A2. Thus, in order to explore the role of eIF5A2 in the doxorubicin-induced EMT, RNAi was applied to knockdown the expression of eIF5A2 in breast cancer cells. The siRNA-transfected breast cancer cells were incubated with doxorubicin or doxorubicin plus GC7 for 48 h. Interestingly, CCK-8 assay revealed that the cell viability in GC7 plus doxorubicin-treated cells (including ERnegative HCC1937 cells) was not significantly different compared to the doxorubicin-treated cells transfected with eIF5A2 siRNA (Fig. 4A-C), suggesting that eIF5A2 was involved in the sensitivity to doxorubicin in ER-negative breast cancer. Western blotting showed the upregulation of E-cadherin and downregulation of vimentin in eIF5A2 siRNA-transfected HCC1937 cells (Fig. 5A and B). However, there were no significant differences in the expression levels of E-cadherin and vimentin in ER-positive MCF-7 and Bcap-37 cells (Fig. 5C and D). In addition, we found that GC7 administration inhibited the hypusination of eIF5A2, 


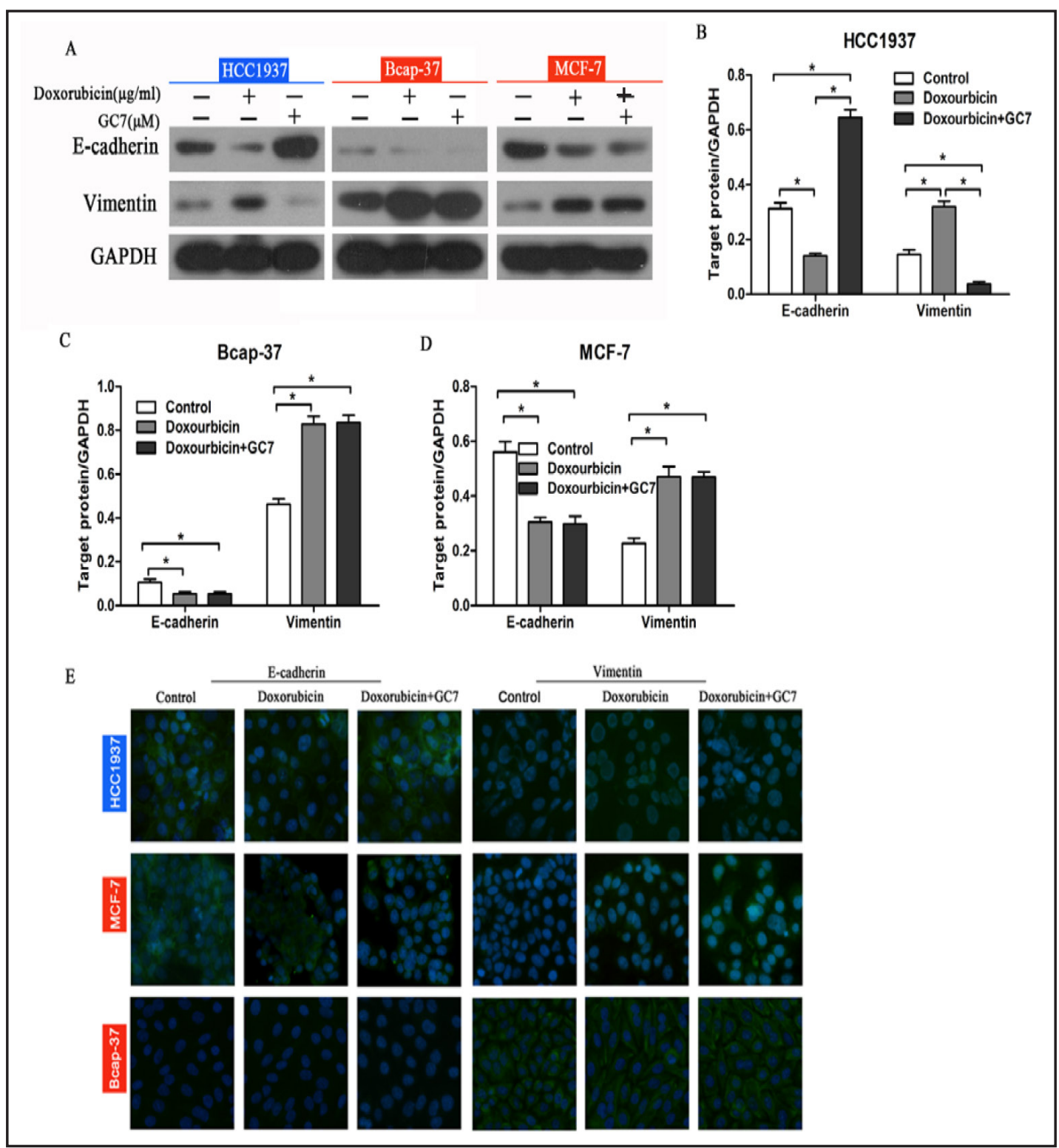

Fig. 3. GC7 treatment altered the expression of doxorubicin-induced EMT-markers in breast cancer cells. Western blotting (A-D) and (E) immunofluorescence were performed to determine the expression of EMT markers E-cadherin and Vimentin in breast cancer HCC1937, Bcap-37 and MCF-7 cells treated with doxorubicin (IC50) alone or doxorubicin plus GC7 $(5 \mu \mathrm{M})$ for $48 \mathrm{~h}$. ${ }^{*} \mathrm{P}<0.05$. All experiments were performed at least three times.

thereby suppressing eIF5A2 activity. But GC7 had no obvious effects on the expression of eIF5A2 (Fig. 5 E). Taken together, these data demonstrated that knockdown of eIF5A2 by siRNA could alter the doxorubicin-induced EMT in ER-negative HCC1937 cells.

\section{Discussion}

It has been long recognized the presence of the ER is considered as a good indicator of disease-free survival and prognosis since patients with ER-positive tumors are candidates for hormonal therapy [18]. In contrast, tumors lacking this receptor have the poorest 
A

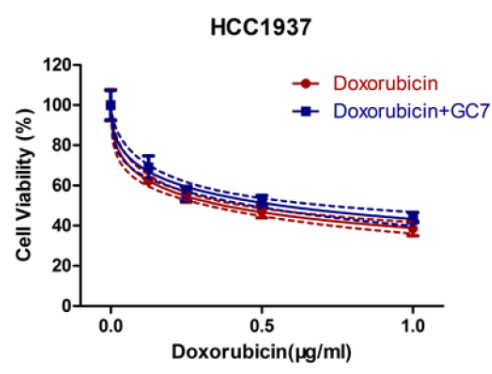

C

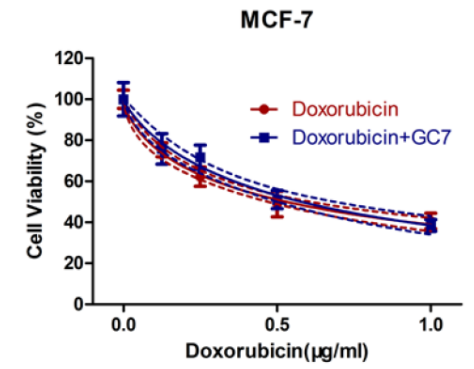

B

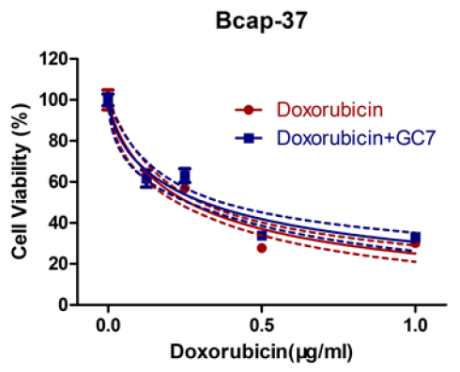

Fig. 4. Cytotoxicity of doxorubicin or doxorubicin plus GC7 in eIF5A2 siRNA-transfected breast cancer cells. Knockdown of eIF5A2 reduced the effect of GC7 plus doxorubicin in ER-negative HCC1937 cells (A), but had little effect in ER-positive MCF-7 (B) and Bcap-37 (C) cells. All experiments were performed at least three times.
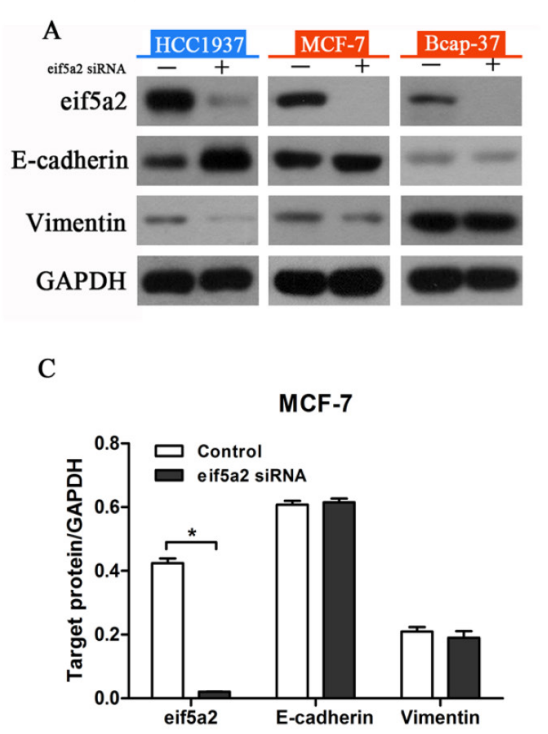

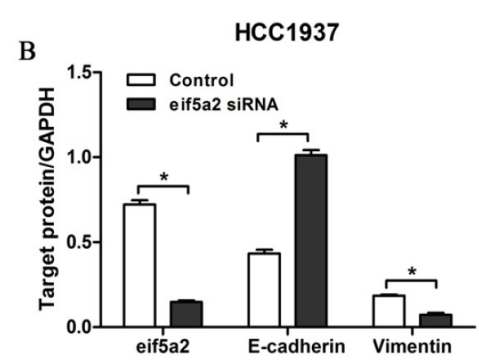

D

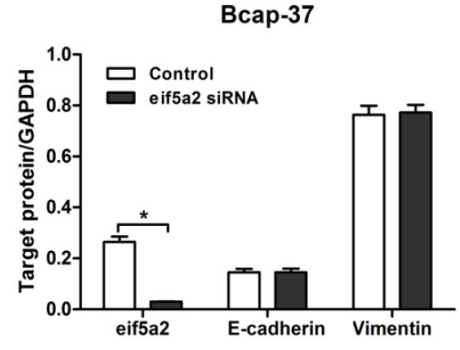

Fig. 5. Knockdown of eIF5A2 reversed the doxorubicin-induced EMT in breast cancer cells. Western blot analysis (A) of E-cadherin and Vimentin expression in eIF5A2-siRNA or Negative-siRNA transfected breast cancer cells treated with doxorubicin for $48 \mathrm{~h}$. Relative protein expression in HCC1937 cells (B), MCF-7 (C) and Bcap-37 (D) cells was quantified by band density with GAPDH served as control. (E) The activity and expression of eIF5A2 were determined with or without GC7 administration in three breast cancer cell lines. $* \mathrm{P}<0.05$. All experiments were performed at least three times.

clinical prognosis often accompanied with resistance to traditional chemotherapeutic drugs [19]. Therefore, combination therapy based on traditional drugs is a promising approach to enhance the effects of chemotherapeutic drugs and relieve the associated adverse side- 
effect. In the present study, we examined whether GC7, an inhibitor of eIF5A2 activation, could enhance the cytotoxicity of doxorubicin in human breast cancer cells. Interestingly, our data showed that GC7 significantly enhanced the chemosensitivity of ER-negative HCC1937 cells to doxorubicin other than ER-positive MCF-7 and Bcap-37 cells.

Exploration of new therapeutics and molecular targets against breast cancer has drawn much attention. Recently, a novel designed cinnamide derivative YLT26 had been shown to induce breast cancer cells apoptosis via mitochondrial apoptotic pathway [20]. In addition, other research groups demonstrated that BAG-1 (bcl-2 associated athanogene) and miR181a could serve as potential therapeutic targets in breast cancer treatment $[21,22]$. It is noteworthy to mention that, to our knowledge, this study is the first to demonstrate the ability of GC7 to sensitize breast cancer cells to doxorubicin in ER-negative breast cancer cells, which we think is of biological importance given its potential therapeutic intervention for ER-negative breast cancer patients.

Epithelial-mesenchymal transition may play a key role in the progression and acquired chemoresistance in many kinds of cancer, including breast cancer [11]. Molecular investigation revealed overexpression of the multi-drug resistance (MDR) gene detected in EMT cancer cells, which may be responsible for the resistance to chemotherapy [23, 24]. Recent studies showed that administration of doxorubicin induced the EMT and then resulted in the resistance to chemotherapy in breast cancer and pancreatic cancer $[13,25]$. Our study found that doxorubicin significantly decreased the expression of E-cadherin, and increased the expression of Vimentin, thus promoting EMT process in all three breast cancer cell lines. However, GC7 treatment reversed the doxorubicin-induced EMT in ER-negative HCC1937 cells, while had little effect on ER-positive MCF-7 and Bcap-37 cells. These results demonstrated that the EMT induced by doxorubicin could be reversed by GC7 in ER-negative breast cancer cells.

Furthermore, we investigated the molecular mechanism underlying the reversal of EMT by GC7. The etiology of breast cancer involves a complex interplay of various factors, of which the accumulation of oncogenes and loss of tumor repressors are crucial events in the initiation and progression of breast cancer [26-28]. Recently studies found that EIF5A2 was aberrantly expressed in several types of tumor cells, including ovarian cancer, bladder cancer, hepatocellular carcinoma and colon cancer [15-16, 29]. Further investigation demonstrated its involvement with the proliferation, invasion, metastasis and chemoresistance of tumor cells $[13,30]$. Thus, eIF5A2, located on chromosome $3 q$, has been characterized as a novel oncogene. In our previous study, we measured eIF-5A2 expression and doxorubicin sensitivity in different human breast cancer cell lines and showed that eIF-5A2 plays an important role in doxorubicin chemoresistance in breast cancer cells [13]. However, the mechanisms of eIF-5A2 regulating this effect are rarely investigated. In the current study, we observed that eIF5A2 was a key factor in doxorubicin-induced EMT in ER-negative breast cancer cells. Moreover, our data showed that knockdown of eIF-5A2 by siRNA could alter the doxorubicin-induced EMT in ER-negative HCC1937 cells.

In conclusion, the present study showed that combined treatment with GC7 enhances the cytotoxicity of doxorubicin in breast cancer cells through inhibiting activation of eIF5A2 and preventing doxorubicin-induced EMT. Therefore, combination therapy with GC7 may contribute to a better therapeutic effect in doxorubicin-based chemotherapy for patients with estrogen receptor-negative breast cancer.

\section{Disclosure Statement}

The authors declare that they have no competing interests.

\section{Acknowledgement}

This study was supported by National 973 Basic Research Program of China (2013CB911303); Traditional Chinese medicine scientific research fund project of Zhejiang 
province (No. 2013ZA076; No.2012ZA079); Zhejiang province natural science fund (No. LY13H160006); co-constructed plan by Ministry of Health and Zhejiang Province (No. 201343278).

\section{References}

1 Siegel R, Naishadham D, Jemal A: Cancer statistics, 2013. CA Cancer J Clin 2013;63:11-30.

2 Engelhardt EG, Garvelink MM, de Haes JH, van der Hoeven JJ, Smets EM, Pieterse AH, Stiggelbout AM: Predicting and communicating the risk of recurrence and death in women with early-stage breast cancer: a systematic review of risk prediction models. J Clin Oncol 2014;32:238-250.

3 Xu C, Kong X, Wang H, Zhang N, Kong X, Ding X, Li X, Yang Q: MTDH mediates estrogen-independent growth and tamoxifen resistance by down-regulating PTEN in MCF-7 breast cancer cells. Cell Physiol Biochem 2014;5:1557-1567.

4 Yang XR, Figueroa JD, Hewitt SM, Falk RT, Pfeiffer RM, Lissowska J, Peplonska B, Brinton LA, Garcia-Closas M, Sherman ME: Estrogen receptor and progesterone receptor expression in normal terminal duct lobular units surrounding invasive breast cancer. Breast Cancer Res Treat 2013;137:837-847.

5 Luo J, Ellis MJ: Microarray data analysis in neoadjuvant biomarker studies in estrogen receptor-positive breast cancer. Breast Cancer Res 2010;12:112.

6 Arumugam A, Subramani R, Nandy S, Lopez R, Boopalan T, Lakshmanaswamy R: Parity and short-term estradiol treatment utilizes similar cellular mechanisms to confer protection against breast cancer. Cell Physiol Biochem 2014;2:491-505.

7 Ford CE, Ekstrom EJ, Anderson T: Retraction for Ford et al., Wnt-5a signaling restores tamoxifen sensitivity in estrogen receptor-negative breast cancer cells. Proc Natl Acad Sci U S A 2010;107:22360-22368.

8 Hartman AR, Kaldate RR, Sailer LM, Painter L, Grier CE, Endsley RR, Griffin M, Hamilton SA, Frye CA, Silberman MA, Wenstrup RJ, Sandbach JF: Prevalence of BRCA mutations in an unselected population of triple-negative breast cancer. Cancer 2012;118:2787-2795.

9 Cellurale C, Girnius N, Jiang F, Cavanagh-Kyros J, Lu S, Garlick DS, Mercurio AM, Davis RJ: Role of JNK in mammary gland development and breast cancer. Cancer Res 2012;72:472-481.

10 Hergovich A: YAP-Hippo signalling downstream of leukemia inhibitory factor receptor: implications for breast cancer. Breast Cancer Res 2012;14:326-329.

11 Al SS, Sharaf LH, Luqmani YA: Signalling pathways involved in endocrine resistance in breast cancer and associations with epithelial to mesenchymal transition (Review). Int J Oncol 2011;38:1197-1217.

12 Clement PM, Johansson HE, Wolff EC, Park MH: Differential expression of eIF5A-1 and eIF5A-2 in human cancer cells. FEBS J 2006;273:1102-1114.

13 Liu Y, Du F, Chen W, Yao M, Lv K, Fu P: EIF5A2 is a novel chemoresistance gene in breast cancer. Breast Cancer 2014;25:135-142.

14 Lee Y, Kim HK, Park MH, Joe YA: Effect of N1-guanyl-1,7-diaminoheptane, an inhibitor of deoxyhypusine synthase, on endothelial cell growth, differentiation and apoptosis. Mol Cell Biochem 2002;237:69-76.

15 Lou B, Fan J, Wang K, Chen W, Zhou X, Zhang J, Lin S, Lv F, Chen Y: N1-guanyl-1,7-diaminoheptane (GC7) enhances the therapeutic efficacy of doxorubicin by inhibiting activation of eukaryotic translation initiation factor 5A2 (eIF5A2) and preventing the epithelial-mesenchymal transition in hepatocellular carcinoma cells. Exp Cell Res 2013;319:2708-2717.

16 Kolligs FT: An alternative way for epithelial-to-mesenchymal transition in colorectal cancer via EIF5A2? Gut 2012;61:473-474.

17 Chehrehasa F, Meedeniya AC, Dwyer P, Abrahamsen G, Mackay-Sim A: EdU, a new thymidine analogue for labelling proliferating cells in the nervous system. J Neurosci Methods 2009;177:122-130.

18 Schott A, Hayes DF: Adjuvant chemotherapy for elderly women with hormone receptor-positive breast cancer: an old(er) problem. J Clin Oncol 2004;22:4660-4662.

19 Kiba T, Inamoto T, Nishimura T, Teramukai S, Kato H, Toi M, Fukushima M: The reversal of recurrence hazard rate between ER positive and negative breast cancer patients with axillary lymph node dissection (pathological stage I-III) 3 years after surgery. BMC Cancer 2008;8:323-331. 
Liu et al.: GC7 Enhances Therapeutic Efficacy of Doxorubicin via eIF5A2

20 Xiong Y, Ye T, Wang M, Xia Y, Wang N, Song X, Wang F, Liu L, Zhu Y, Yang F,Wei Y, Yu L: A novel cinnamide YLT26 induces breast cancer cells apoptosis via ROS-mitochondrial apoptotic pathway in vitro and inhibits lung metastasis in vivo. Cell Physiol Biochem 2014;6:1863-1876.

21 Liu H, Lu S, Gu L, Gao Y, Wang T, Zhao J, Rao J, Chen J, Hao X, Tang SC: Modulation of BAG-1 expression alters the sensitivity of breast cancer cells to tamoxifen. Cell Physiol Biochem 2014;2:365-374.

22 Zhu Y, Wu J, Li S, Ma R, Cao H, Ji M, Jing C, Tang J. The function role of miR-181a in chemosensitivity to adriamycin by targeting Bcl-2 in low-invasive breast cancer cells. Cell Physiol Biochem 2013; 5:1225-1237.

23 Li QQ Xu JD, Wang WJ, Cao XX, Chen Q Tang F, Chen ZQ, Liu XP, Xu ZD: Twist1-mediated adriamycininduced epithelial-mesenchymal transition relates to multidrug resistance and invasive potential in breast cancer cells. Clin Cancer Res 2009;15:2657-2665.

24 Saxena M, Stephens MA, Pathak H, Rangarajan A: Transcription factors that mediate epithelialmesenchymal transition lead to multidrug resistance by upregulating ABC transporters. Cell Death Dis 2011;2:179-183.

25 Rhim AD, Mirek ET, Aiello NM, Maitra A, Bailey JM, McAllister F, Reichert M, Beatty GL, Rustgi AK, Vonderheide RH, Leach SD, Stanger BZ: EMT and dissemination precede pancreatic tumor formation. Cell 2012;148:349-361.

26 Zhao H, Lo YH, Yu L, Wang SC: Overcoming resistance to fulvestrant (ICI182,780) by downregulating the c-ABL proto-oncogene in breast cancer. Mol Carcinog 2011;50:383-389.

27 Wajapeyee N, Malonia SK, Green MR: Oncogenic RAS directs silencing of tumor suppressor genes through ordered recruitment of transcriptional repressors. Genes Dev 2013;27:2221-2226.

28 Azhar M: Non-redundant tumour supressor functions of transforming growth factor beta in breast cancer. J Biosci 2001;26:9-12.

29 Yang GF, Xie D, Li LJ, Hua WF, Wu HM, Kung HF, Zeng YX, Guan XY: Expression and amplification of eIF-5A2 in human epithelial ovarian tumors and overexpression of EIF-5A2 is a new independent predictor of outcome in patients with ovarian carcinoma. Gynecol Oncol 2009;112:314-318.

30 Li Y, Fu L, Li JB, Qin Y, Zeng TT, Zhou J, Zeng ZL, Chen J, Cao TT, Ban X, Qian C, Cai Z, Xie D, Huang P, Guan XY: Increased Expression of EIF5A2, Via Hypoxia or Gene Amplification, Contributes to Metastasis and Angiogenesis of Esophageal Squamous Cell Carcinoma. Gastroenterology 2014;146:1701-1713. 